\title{
The Influence of Partial Isomorphic Substitution on Electronic and Optical Parameters of $\mathrm{ABSO}_{4}$ Group Crystals
}

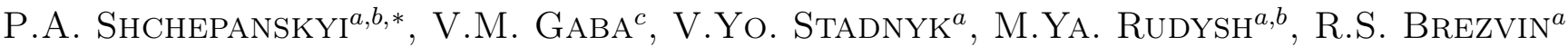 \\ AND M. PIASECKI ${ }^{b}$ \\ ${ }^{a}$ Faculty of Physics, Ivan Franko National University of Lviv, 8 Kyrylo-and-Mefodii Str., UA-79005 Lviv, Ukraine \\ ${ }^{b}$ Institute of Physics, Jan Dlugosz Academy, Armii Krajowej 13/15, PL-42201, Czestochowa, Poland \\ ${ }^{c}$ National University "Lviv Politechnic", 5 Mitropolit Andrei Str., UA-79013 Lviv, Ukraine
}

\begin{abstract}
First principles calculations of band energy, density of states and dielectric function of $\mathrm{K}_{1.75}\left(\mathrm{NH}_{4}\right)_{0.25} \mathrm{SO}_{4}$ single crystals in Pnma structure is performed in the framework of density functional theory. In order to describe the exchange-correlation interaction the general gradient approximation and local density approximation are used. Character and genesis of main energy bands of the crystal are discussed and comparison with data set for $\mathrm{K}_{2} \mathrm{SO}_{4}$ isomorphic crystal performed. The influence of partial isomorphic substitution $\mathrm{K} \rightarrow \mathrm{NH}_{4}$ found itself mainly in altering the bottom of conduction band and decrease of band gap value. From the dielectric functions dependences, the refractive indices dispersion and absorption coefficient spectra are deduced. Obtained refractive indices satisfactorily agree with experimental data in the visible spectrum region.
\end{abstract}

DOI: 10.12693/APhysPolA.133.819

PACS/topics: 71.20.-b, 77.84.-s, 77.84.Bw

\section{Introduction}

Crystals of $\mathrm{ABSO}_{4}$ group, where $\mathrm{A}$ and $\mathrm{B}$ are alkali metal cations, are of a significant interest due to ability to undergo a richness of phase transitions, their ferroelectric, ferroelastic, superionic, and optical properties. Among the last mentioned ones interesting feature of $\mathrm{ABSO}_{4}$ group crystals is possibility of existing of isotropic points, when crystal undergoes transition from biaxial to uniaxial or from uniaxial to isotropic. Such crystals can be used as temperature and pressure sensors $[1,2]$.

Lately, with an aim of revealing the influence of partial isomorphic substitution $\mathrm{K} \rightarrow \mathrm{NH}_{4}$ on optical properties of crystals, the $\mathrm{K}_{1.75}\left(\mathrm{NH}_{4}\right)_{0.25} \mathrm{SO}_{4}$ single crystal was studied [3]. This one is representative of $\mathrm{K}_{2} \mathrm{SO}_{4}{ }^{-}$ $\left(\mathrm{NH}_{4}\right)_{2} \mathrm{SO}_{4}$ system, which is known to form a continuous series of solid solutions with $\left(\mathrm{K}_{x}\left(\left.\mathrm{NH}_{4}\right|_{1-x}\right)_{2} \mathrm{SO}_{4}\right.$, $(0<x<1)$ general formula [4].

$\mathrm{K}_{1.75}\left(\mathrm{NH}_{4}\right)_{0.25} \mathrm{SO}_{4}$ crystal belongs to Pnma (No.62) space group. Its lattice parameters are equal to: $a=$ $7.5562(3) \AA, b=5.7917(2) \AA, c=10.1016(4) \AA$, cell volume $=442.08(5) \AA^{3}[3]$. Among the peculiarities of $\mathrm{K}_{1.75}\left(\mathrm{NH}_{4}\right)_{0.25} \mathrm{SO}_{4}$ crystals optical properties the abnormal behavior of birefringence dispersion in the direction of perpendicular to optical axes plane, leading to the existence of isotropic point at room temperature not observable for either $\mathrm{K}_{2} \mathrm{SO}_{4}$ nor $\left(\mathrm{NH}_{4}\right)_{2} \mathrm{SO}_{4}$ was found. Such point was reported to occur at the wavelength $\approx 1350 \mathrm{~nm}$. Considering that $\mathrm{K}_{1.75}\left(\mathrm{NH}_{4}\right)_{0.25} \mathrm{SO}_{4}$ crystal is relatively

*corresponding author; e-mail: pavloshchepanskyi@gmail.com mechanically stable and has no phase transitions in a wide temperature region, it was found to be appropriate for practical implementation in crystal optics sensing.

Expanding mentioned study interesting is to investigate the influence of partial isomorphic substitution on electronic properties of crystals and also simulate the optical properties from first principles. Since band structures for $\mathrm{K}_{2} \mathrm{SO}_{4}$ [5] crystal is known as well as for other isomorphic crystals of that family, desirable peculiarities can be revealed. Knowing the character of properties changing can help in predicting the most appropriate candidate for practical application of this system.

Thus in this work calculations of band structure, density of states and refractive indices spectra of the $\mathrm{K}_{1.75}\left(\mathrm{NH}_{4}\right)_{0.25} \mathrm{SO}_{4}$ crystals within the density functional theory (DFT) are performed.

\section{Calculation technique}

Calculations of the band structure were carried out self-consistently using the Kohn-Sham formalism within density functional theory (DFT). As input parameters the X-ray analysis data was used [3]. Basis was chosen in a form of plane waves. To describe the exchangecorrelation interaction the exchange-correlation functionals with the generalized gradient approximation (GGA) and local density approximation (LDA) were used. The electron-ion interaction was taken into account as the Vanderbilt ultrasoft pseudopotential. Electronic configuration is $\mathrm{H}\left(1 s^{1}\right), \mathrm{N}\left(2 s^{2} 2 p^{3}\right), \mathrm{O}\left(2 s^{2} 2 p^{4}\right), \mathrm{S}\left(3 s^{2} 3 p^{4}\right)$, $\mathrm{K}\left(3 s^{2} 3 p^{6} 4 s^{1}\right)$.

Convergence processes aiming to find the optimal calculation parameters are presented in Fig.1. Since total energy for two neighboring convergence points do not differ $E_{\text {total }}(380)=E_{\text {total }}(400)=-13908.09 \mathrm{eV}$, the maximal kinetic energy of plane waves was limited by the 
value of $380 \mathrm{eV}$. In the similar way we have chosen the Monkhorst-Pack $2 \times 2 \times 1$ grid for integration over $\boldsymbol{k}$ points of the Brillouin zone (BZ) $\left(E_{\text {total }}(2 \times 2 \times 1)=\right.$ $\left.E_{\text {total }}(2 \times 3 \times 2)=-13907.31 \mathrm{eV}\right)$, though $2 \times 3 \times 2$ and $3 \times 4 \times 3$ grids were also employed for comparison. Calculations of optical parameters were carried out conventionally using larger grid, namely $3 \times 4 \times 2$.
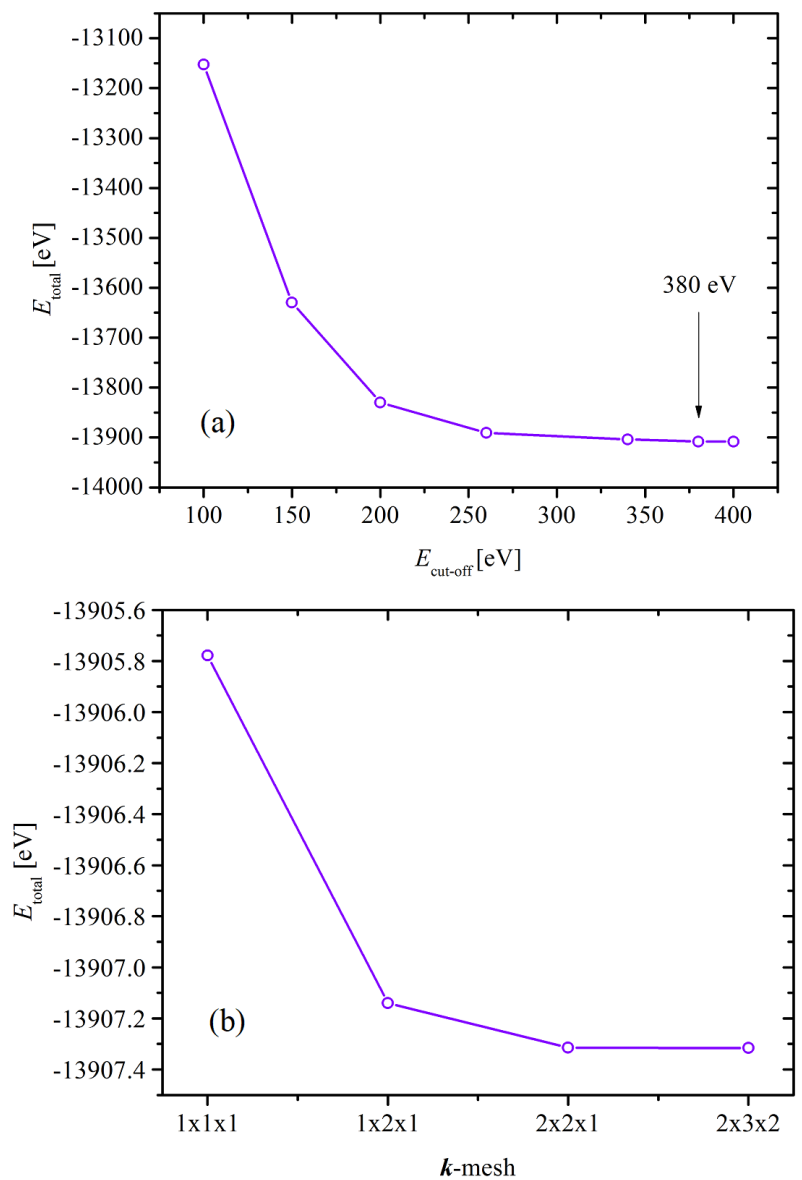

Fig. 1. Cut-off energy (a) and $\boldsymbol{k}$-mesh (b) convergences for the $\mathrm{K}_{1.75}\left[\mathrm{NH}_{4}\right]_{0.25} \mathrm{SO}_{4}$ crystal.

Unit cell and position of atoms were optimized before calculations using the Broyden, Fletcher, Goldfarb and Shanno (BFGS) algorithm. Parameters used for calculations were chosen as follows: criterion for stopping the self-consistency cycle was reaching the convergence with accuracy $E=5.0 \times 10^{-7} \mathrm{eV}$ per atom; as criteria for obtaining structural geometry of good quality next parameters were used: convergence of the total energy $5.0 \times 10^{-6} \mathrm{eV}$ per atom, maximum force $0.01 \mathrm{eV} / \AA$, maximum pressure $0.02 \mathrm{GPa}$, maximum displacement $5.0 \times 10^{-4} \AA$.

Structure of the Brillouin zone used for building of the band energy diagram contained special points with next coordinates: $\Gamma(0 ; 0 ; 0), Z(0 ; 0 ; 0.5), \quad T(0 ; 0.5 ; 0.5)$, $Y(0 ; 0.5 ; 0), \quad S(0.5 ; 0.5 ; 0), \quad X(0.5 ; 0 ; 0), \quad U(0.5 ; 0 ; 0.5)$, $R(0.5 ; 0.5 ; 0.5)$

\section{Results and discussion}

\subsection{Electronic structure calculations}

Experimentally obtained in work [3] unit cell parameters and optimized using BFGS method as well as coordinates of atoms are presented in Tables I and II. One can see that calculated parameters for GGA are slightly higher $(\Delta a \approx 5.4 \%, \Delta b \approx 0.7 \%, \Delta c \approx 3.5 \%$ ) but close to experimental. Using LDA functional gives relatively better agreement with experiment $(\Delta a \approx 3.3 \%, \Delta b \approx 1.7 \%$, $\Delta c \approx 1.4 \%$ ). Optimized volume of unit cell is greater in comparison with experiment for about $9.3 \%$ for GGA and $6.6 \%$ for LDA.

TABLE I

The atomic positions for the $\mathrm{K}_{1.75}\left[\mathrm{NH}_{4}\right]_{0.25} \mathrm{SO}_{4}$ crystal.

\begin{tabular}{c|c|c|c|c}
\hline \hline Atom & Wyckoff & \multicolumn{3}{|c}{ Optimized coordinates of atoms } \\
\cline { 3 - 5 } & positions & $x / a$ & $y / b$ & $z / c$ \\
\hline $\mathrm{H} 1$ & $4 c$ & 0.686226 & 0.072418 & 0.076795 \\
$\mathrm{H} 2$ & $4 c$ & 0.686881 & 0.335728 & 0.003450 \\
$\mathrm{H} 3$ & $4 c$ & 0.566721 & 0.290826 & 0.134645 \\
$\mathrm{H} 4$ & $4 c$ & 0.778447 & 0.298923 & 0.149497 \\
$\mathrm{~N} 1$ & $4 c$ & 0.680000 & 0.250000 & 0.091300 \\
$\mathrm{O} 1$ & $8 d$ & 0.300000 & 0.040400 & 0.144500 \\
$\mathrm{O} 2$ & $4 c$ & 0.200000 & 0.959600 & 0.644500 \\
$\mathrm{O} 3$ & $4 c$ & 0.700000 & 0.540400 & 0.855500 \\
$\mathrm{O} 4$ & $4 c$ & 0.800000 & 0.459600 & 0.355500 \\
$\mathrm{O} 5$ & $8 d$ & 0.700000 & 0.959600 & 0.855500 \\
$\mathrm{O} 6$ & $4 c$ & 0.800000 & 0.040400 & 0.355500 \\
$\mathrm{O} 7$ & $4 c$ & 0.300000 & 0.459600 & 0.144500 \\
$\mathrm{O} 8$ & $4 c$ & 0.200000 & 0.540400 & 0.644500 \\
$\mathrm{O} 9$ & $8 d$ & 0.050000 & 0.250000 & 0.089000 \\
$\mathrm{O} 10$ & $4 c$ & 0.790000 & 0.250000 & 0.556700 \\
$\mathrm{O} 11$ & $4 c$ & 0.450000 & 0.750000 & 0.589000 \\
$\mathrm{O} 12$ & $4 c$ & 0.710000 & 0.750000 & 1.056700 \\
$\mathrm{O} 13$ & $8 d$ & 0.950000 & 0.750000 & 0.911000 \\
$\mathrm{O} 14$ & $4 c$ & 0.210000 & 0.750000 & 0.443300 \\
$\mathrm{O} 15$ & $4 c$ & 0.550000 & 0.250000 & 0.411000 \\
$\mathrm{O} 16$ & $4 c$ & 0.290000 & 0.250000 & -0.056700 \\
$\mathrm{~S} 1$ & $4 c$ & 0.235200 & 0.250000 & 0.078400 \\
$\mathrm{~S} 2$ & $4 c$ & 0.264800 & 0.750000 & 0.578400 \\
$\mathrm{~S} 3$ & $4 c$ & 0.764800 & 0.750000 & 0.921600 \\
$\mathrm{~S} 4$ & $4 c$ & 0.735200 & 0.250000 & 0.421600 \\
$\mathrm{~K} 1$ & $4 c$ & 0.180000 & 0.250000 & 0.408700 \\
$\mathrm{~K} 2$ & $4 c$ & 0.486900 & 0.250000 & 0.702900 \\
$\mathrm{~K} 3$ & $4 c$ & 0.320000 & 0.750000 & 0.908700 \\
$\mathrm{~K} 4$ & $4 c$ & 0.013100 & 0.750000 & 0.202900 \\
$\mathrm{~K} 5$ & $4 c$ & 0.820000 & 0.750000 & 0.591300 \\
$\mathrm{~K} 6$ & $4 c$ & 0.513100 & 0.750000 & 0.297100 \\
$\mathrm{~K} 7$ & $4 c$ & 0.986900 & 0.250000 & 0.797100 \\
& & & &
\end{tabular}

Parameters of the band structure calculated with two sets of grids are presented in Table III. Since the values obtained with larger grid do not reveal significant difference from those obtained using $2 \times 2 \times 1$ grid, the latter is used for band structure analysis in this work. 
TABLE II

Lattice parameters $a, b, \quad c$ and volume $V$ of $\mathrm{K}_{1.75}\left(\mathrm{NH}_{4}\right)_{0.25} \mathrm{SO}_{4}$ crystal, obtained experimentally [3] and from first principles. Space group Pnma (No. 62), $Z=4$.

\begin{tabular}{c|c|c|c}
\hline \hline Compound: & \multirow{2}{*}{ Experimental } & \multicolumn{2}{|c}{ Calculated } \\
\cline { 3 - 4 } $\mathrm{K}_{1.75}\left[\mathrm{NH}_{4}\right]_{0.25} \mathrm{SO}_{4}$ & & GGA & LDA \\
\hline$a[\AA]$ & $7.5562(3)$ & 7.9850 & 7.8085 \\
$b[\AA]$ & $5.7917(2)$ & 5.8303 & 5.6950 \\
$c[\AA]$ & $10.1016(4)$ & 10.4729 & 10.2442 \\
$c / a$ & 1.33686 & 1.3116 & 1.3119 \\
$c / b$ & 1.74415 & 1.7963 & 1.7988 \\
volume $\left[\AA^{3}\right]$ & $442.08(5)$ & 487.5569 & 455.5535
\end{tabular}

TABLE III

Band energy structure parameters of $\mathrm{K}_{1.75}\left(\mathrm{NH}_{4}\right)_{0.25} \mathrm{SO}_{4}$ crystal obtained with $2 \times 2 \times 1,2 \times 3 \times 2$ and $3 \times 4 \times 3$ $\boldsymbol{k}$-mesh grids.

\begin{tabular}{c|c|c|c|c|c|c}
\hline \hline \multirow{2}{*}{$\begin{array}{c}\text { Point of } \\
\text { Brillouin zone }\end{array}$} & \multicolumn{2}{|c|}{$2 \times 2 \times 1$} & \multicolumn{2}{c|}{$2 \times 3 \times 2$} & \multicolumn{2}{c}{$3 \times 4 \times 3$} \\
\cline { 2 - 7 } & $\varepsilon_{c}$ & $\varepsilon_{v}$ & $\varepsilon_{c}$ & $\varepsilon_{v}$ & $\varepsilon_{c}$ & $\varepsilon_{v}$ \\
\hline$\Gamma(0 ; 0 ; 0)$ & 4.80 & 0 & 4.81 & 0 & 4.81 & 0 \\
\hline$Z(0 ; 0 ; 0.5)$ & 5.36 & -0.01 & 5.36 & -0.01 & 5.36 & -0.01 \\
\hline$T(0 ; 0.5 ; 0.5)$ & 6.11 & -0.05 & 6.21 & -0.07 & 6.20 & -0.07 \\
\hline$Y(0 ; 0.5 ; 0)$ & 5.74 & -0.05 & 6.05 & -0.07 & 6.03 & -0.07 \\
\hline$S(0.5 ; 0.5 ; 0)$ & 6.82 & -0.14 & 6.82 & -0.14 & 6.82 & -0.14 \\
\hline$X(0.5 ; 0 ; 0)$ & 6.26 & -0.08 & 5.93 & -0.06 & 5.95 & -0.07 \\
\hline$U(0.5 ; 0 ; 0.5)$ & 6.41 & -0.08 & 6.40 & -0.05 & 6.40 & -0.06 \\
\hline$R(0.5 ; 0.5 ; 0.5)$ & 6.88 & -0.14 & 6.89 & -0.14 & 6.88 & -0.14
\end{tabular}

Calculated band energy diagrams for $\mathrm{K}_{1.75}\left(\mathrm{NH}_{4}\right)_{0.25} \mathrm{SO}_{4}$ crystal using GGA and LDA functionals are presented in Figs. 2 and 3, respectively. Significant differences between obtained diagrams are not detected. As can be seen from the figures, almost all energy levels are of a low dispersion. The exception make the lowest conduction band (CB) levels of the BZ divisions $\Gamma-Z$ and $\Gamma-F$, where dispersion dependence $E(k)$ is maximal. Observable characteristics was also revealed for such crystals as $\mathrm{K}_{2} \mathrm{SO}_{4}$ [5], $\mathrm{RbNH}_{4} \mathrm{SO}_{4}$ [6], $\mathrm{LiRbSO}_{4}$ [7], $\alpha-\mathrm{LiNH}_{4} \mathrm{SO}_{4} \quad[8]$ and $\beta-\mathrm{LiNH}_{4} \mathrm{SO}_{4} \quad[9]$ and thus can be qualified as typical for $\mathrm{ABSO}_{4}$ type crystals. Interesting feature of $\mathrm{K}_{1.75}\left(\mathrm{NH}_{4}\right)_{0.25} \mathrm{SO}_{4}$ crystals band structure is visible "splitting" of lowest $\mathrm{CB}$ levels not observable for $\mathrm{K}_{2} \mathrm{SO}_{4}$ crystal or any studied of this group.

Top of valence band (VB), marked as $0 \mathrm{eV}$, coincide with the Fermi level $E_{F}$. The energy band gap of the $\mathrm{K}_{1.75}\left(\mathrm{NH}_{4}\right)_{0.25} \mathrm{SO}_{4}$ crystal is of a direct type and corresponds to the optical transitions in the $\Gamma$ point of the BZ (Figs. 2 and 3). Obtained $E_{g}=4.80 \mathrm{eV}$ (GGA) and $E_{g}=4.89 \mathrm{eV}(\mathrm{LDA})$ values for the crystal are expectedly lower then calculated for $\mathrm{K}_{2} \mathrm{SO}_{4}[5]\left(E_{g}=5.2 \mathrm{eV}\right)$ and lie between $4.5 \mathrm{eV}$ energy of optical long-wave absorption edge of the $\left(\mathrm{NH}_{4}\right)_{2} \mathrm{SO}_{4}$ crystal [10] and $7.2 \mathrm{eV}$ energy of similar edge for the $\mathrm{K}_{2} \mathrm{SO}_{4}$ crystal [11]. However they are for about $2 \mathrm{eV}$ underestimated due to known features

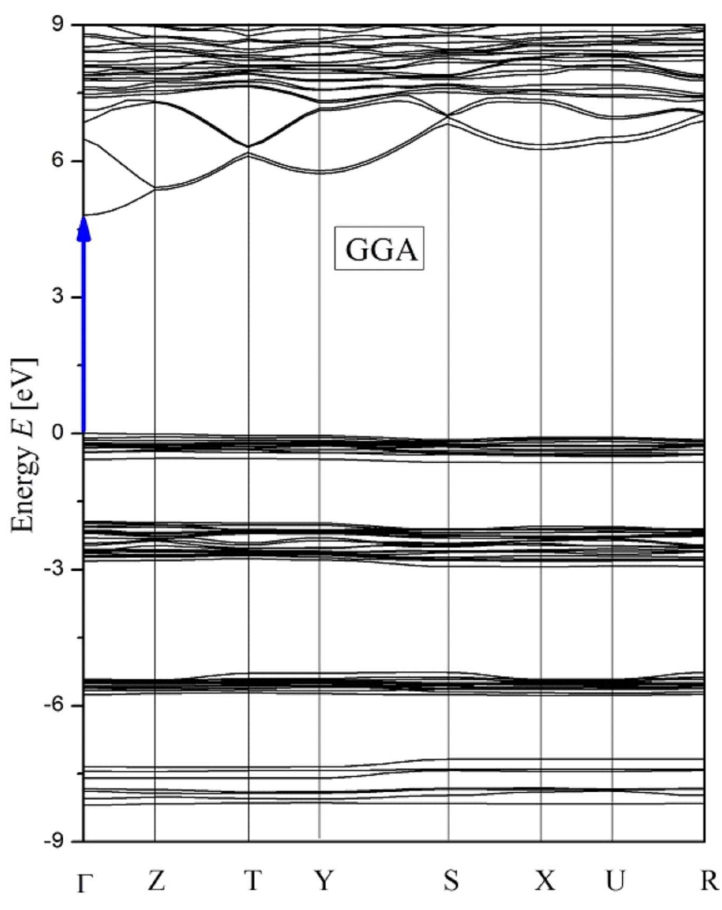

Fig. 2. Calculated band energy diagram for $\mathrm{K}_{1.75}\left(\mathrm{NH}_{4}\right)_{0.25} \mathrm{SO}_{4}$ crystal for the GGA functional $\left(E_{g}=4.80 \mathrm{eV}\right)$.

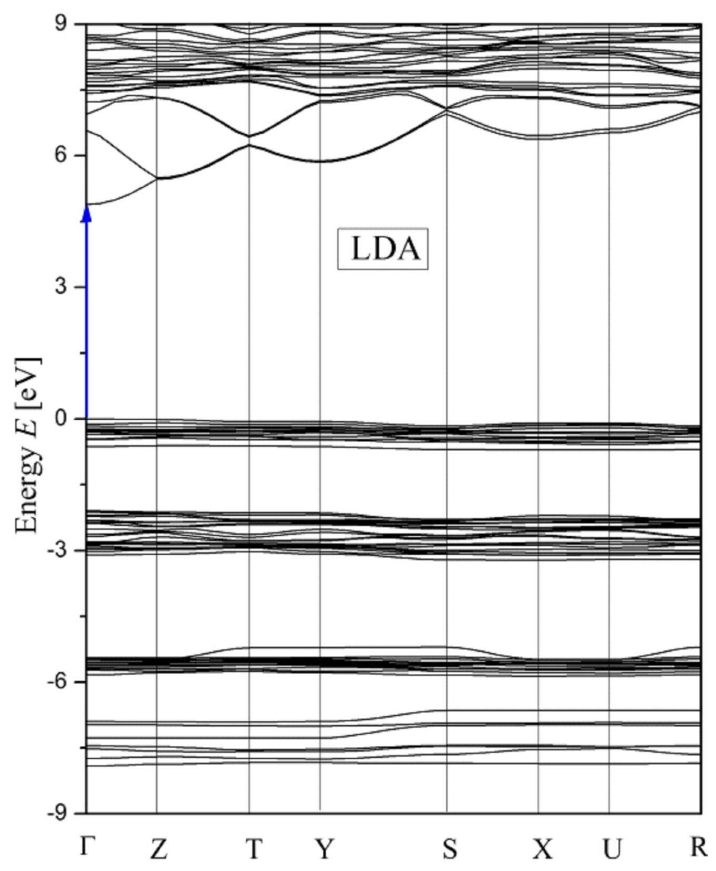

Fig. 3. Calculated band energy diagram for $\mathrm{K}_{1.75}\left(\mathrm{NH}_{4}\right)_{0.25} \mathrm{SO}_{4}$ crystal for the LDA functional $\left(E_{g}=4.89 \mathrm{eV}\right)$.

of DFT method. Obtained results allow us to assume that the fundamental absorption edge is associated with direct transitions between the top of VB and the bottom of $\mathrm{CB}$ at $\Gamma$ point, the center of the Brillouin zone.

Total density of electronic states $N(E)$ and partial density of states (PDOS), with the contributions of individ- 
ual atoms, are calculated for band-energy diagram $E(k)$ of $\mathrm{K}_{1.75}\left(\mathrm{NH}_{4}\right)_{0.25} \mathrm{SO}_{4}$ crystal (Fig. 4). As can be seen from the figure, a characteristic feature of PDOS is that two top VBs (from -3.1 to -1.3 and from -1.2 to $0 \mathrm{eV}$ ) are almost entirely formed by $p$-electrons of oxygen from $\mathrm{SO}_{4}^{2-}$ anion complexes. An insignificant contribution to these two bands gives $p$-states of sulfur and nitrogen and $s$-electrons of hydrogen. Deeper levels of the valence band in the range from -4.8 to $-8 \mathrm{eV}$ are of a mixed origin and are formed by $s$ - and $p$-states of sulfur and oxygen as well as $s$-states of hydrogen hybridized with $p$-states of nitrogen. States of potassium of a low intensity are also present here.

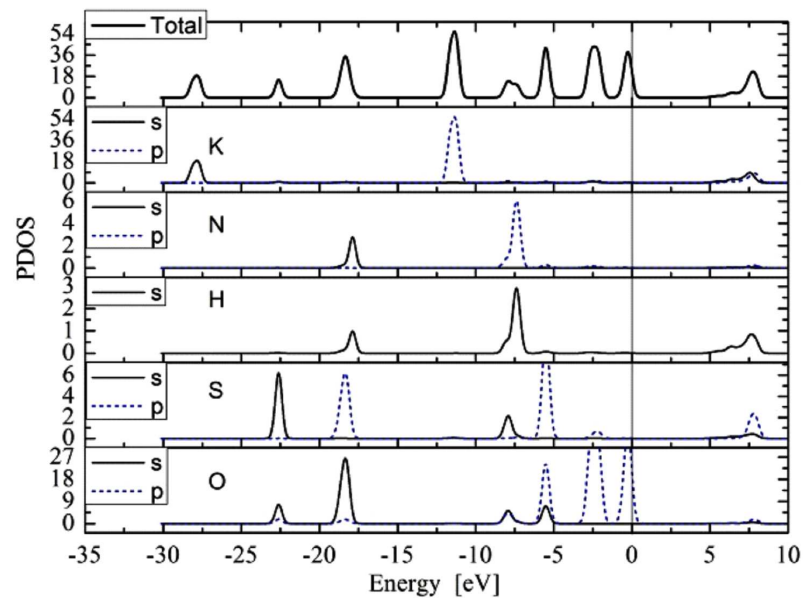

Fig. 4. Total and partial density of electronic states for band-energy diagram $E(\boldsymbol{k})$ of $\mathrm{K}_{1.75}\left(\mathrm{NH}_{4}\right)_{0.25} \mathrm{SO}_{4}$ crystal.

Valence bands from -17.5 to $-19 \mathrm{eV}$ contain two similar sub-zones, one of which is formed by $\mathrm{NH}_{4}$ group $(s$ states of nitrogen and hydrogen), and another - by $\mathrm{SO}_{4}$ cationic complexes ( $s$-states of oxygen hybridized with $p$ states of sulfur). Energy levels near $-22.5 \mathrm{eV}$ are formed mainly by $s$-states of sulfur $\mathrm{S}$ and oxygen O. Contribution of potassium atoms electronic states is major for $\mathrm{VB}$ at $-28 \mathrm{eV}$.

The bottom of the $\mathrm{CB}$ is formed mainly by hydrogen atoms ( $s$-states); higher areas of this band at $E>6.1 \mathrm{eV}$ - by mixed set of chemical elements and orbital moments.

Obtained band structure of the $\mathrm{K}_{1.75}\left(\mathrm{NH}_{4}\right)_{0.25} \mathrm{SO}_{4}$ crystal is quite similar to calculated for $\mathrm{K}_{2} \mathrm{SO}_{4}$ [5]. An exception makes the fact that bottom of $\mathrm{CB}$ in $\mathrm{K}_{1.75}\left(\mathrm{NH}_{4}\right)_{0.25} \mathrm{SO}_{4}$ is formed by states of hydrogen, while similar role in $\mathrm{K}_{2} \mathrm{SO}_{4}$ is played by potassium states. This leads to decrease of band gap in the mixed crystal for about $0.4 \mathrm{eV}$ in comparison with PS. Study of PDOS have also helped to reveal that observable "splitting" in the bottom of CB is caused by nearby situated potassium and hydrogen states.

\subsection{Optical properties}

Optical properties of materials can be studied by investigation of complex dielectric function $\varepsilon(\omega)$. Its imag- inary part $\varepsilon_{2}(\omega)$ can be calculated from the momentum matrix elements between the occupied and unoccupied wave-functions [12]:

$$
\begin{aligned}
& \varepsilon_{2}(\omega)=\left.\frac{2 \pi \mathrm{e}^{2}}{\Omega \varepsilon_{0}} \sum_{k, v, c}\left|\left\langle\psi_{k}^{c}|\boldsymbol{u} \boldsymbol{r}|\right\rangle \psi_{k}^{v}\right\rangle\right|^{2} \\
& \quad \times \delta\left(E_{k}^{c}-E_{k}^{v}-E\right),
\end{aligned}
$$

where $\boldsymbol{u}$ is polarization vector of incident photon; $\psi_{k}^{c}$ and $\psi_{k}^{v}$ are the $\mathrm{CB}$ and $\mathrm{VB}$ wave-functions in $\boldsymbol{k}$-space, and $\boldsymbol{r}$ is electron position operator.

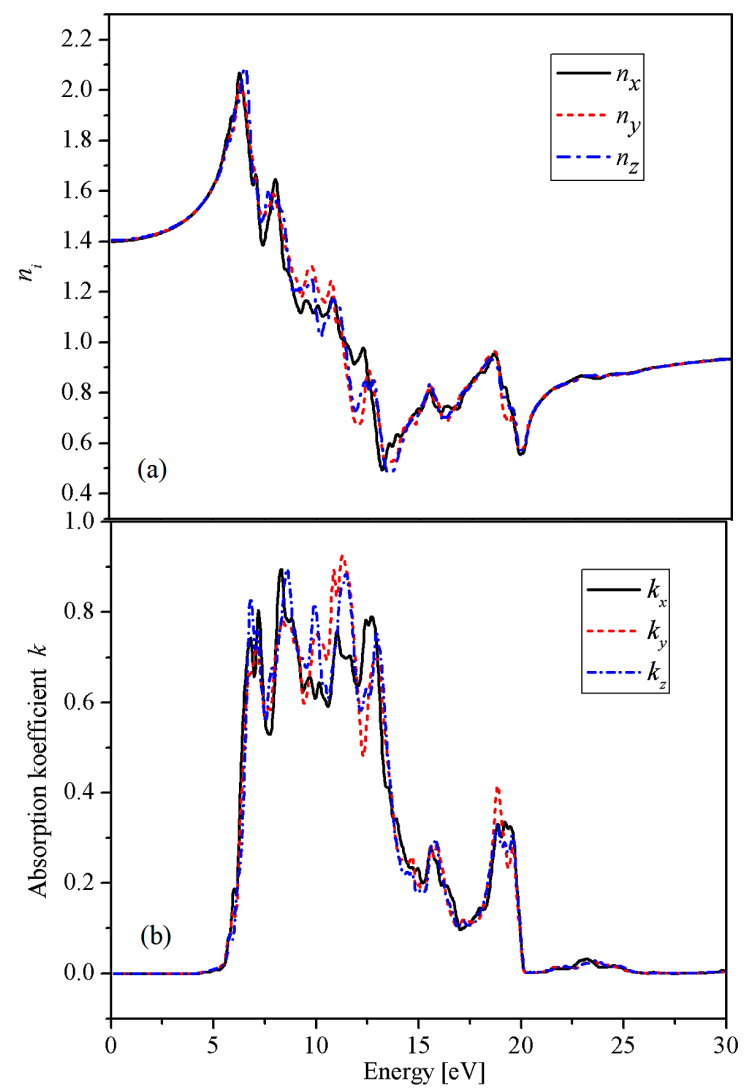

Fig. 5. Refractive indices (a) and absorption (b) spectra for $\mathrm{K}_{1.75}\left(\mathrm{NH}_{4}\right)_{0.25} \mathrm{SO}_{4}$ crystal for three principal crystal optics directions (GGA).

The real part $\varepsilon_{1}(\omega)$ of the dielectric function can be evaluated from the imaginary part $\varepsilon_{2}(\omega)$ by the wellknown Kramers-Kronig relation

$$
\varepsilon_{1}(\omega)=1+\frac{2}{\pi} \int_{0}^{\infty} \frac{\varepsilon_{2}\left(\omega^{\prime}\right) \omega^{\prime} \mathrm{d} \omega^{\prime}}{\omega^{\prime 2}-\omega^{2}} .
$$

From the spectrum of real and imaginary parts of dielectric function, refractive indices $n(\omega)$ and absorption coefficients $k(\omega)$ can be obtained using the relations [13]:

$$
n=\sqrt{\frac{\left(\varepsilon_{1}^{2}+\varepsilon_{2}^{2}\right)^{\frac{1}{2}}+\varepsilon_{1}}{2}}, \quad k=\sqrt{\frac{\left(\varepsilon_{1}^{2}+\varepsilon_{2}^{2}\right)^{\frac{1}{2}}-\varepsilon_{1}}{2}} .
$$

Calculated frequency dependences of refractive indices along with absorption coefficients spectra for the 


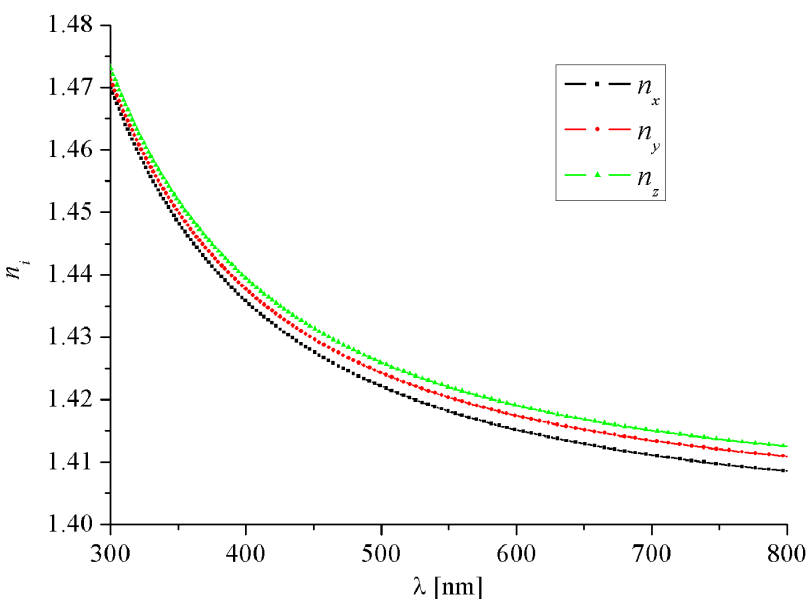

Fig. 6. Dispersion of refractive indices calculated for three principal crystal optics directions of $\mathrm{K}_{1.75}\left(\mathrm{NH}_{4}\right)_{0.25} \mathrm{SO}_{4}$.

$\mathrm{K}_{1.75}\left(\mathrm{NH}_{4}\right)_{0.25} \mathrm{SO}_{4}$ single crystal are presented in Fig. 5. Zero frequency values of refractive indices are $n_{z}(0)=$ $1.405, n_{x}(0) \approx 1.400, n_{y}(0)=1.403$. The most considerable anisotropic behavior is found between the $z$ and $x$ components.

Refractive indices dispersions for the $\mathrm{K}_{1.75}\left(\mathrm{NH}_{4}\right)_{0.25} \mathrm{SO}_{4}$ are presented for the visible spectrum region in Fig. 6. As can be seen from the figure, $n_{i}$ satisfy the relation $n_{z}>n_{x}>n_{y}$ on the whole presented region. Order of obtained curves coincides with experimental one [3]. Values of refractive indices are for about $5.5 \%$ lower than experimental ones. The difference is also that dispersion of theoretically obtained curves is of the same magnitude for three directions of crystal, while magnitudes for experimental dependences slightly differ, leading to the crossing $n_{x}=n_{y}$ in the near IR spectrum region. Such discrepancies between first principles and experimental results are assumed to occur due to neglect of IR absorption of crystal in the calculation and presence of defects in real crystals.

\section{Conclusions}

Performed first principle calculations of electronic and optical properties of $\mathrm{K}_{1.75}\left(\mathrm{NH}_{4}\right)_{0.25} \mathrm{SO}_{4}$ single crystals in Pnma structure allow to make following conclusions.

Optimized before calculations crystal structure is close to obtained experimentally. All levels except the lowest ones of $\mathrm{CB}$ in the direction towards the center of BZ reveal low dispersion of electronic states $E(\boldsymbol{k})$. The energy band gap of the crystal is of a direct type and corresponds to the optical transitions in the $\Gamma$ point of the BZ with $E_{g}=4.80 \mathrm{eV}$ (GGA) and $E_{g}=4.89 \mathrm{eV}$ (LDA). The top of the VB is formed by $p$-electrons of oxygen. The bottom of the CB is formed mainly by $s$-states of hydrogen atoms.

In general, partial isomorphic substitution $\mathrm{K} \rightarrow \mathrm{NH}_{4}$ $(12.5 \%)$ is found to alter the band structure with changing the bottom levels of conduction band origin (potassium $s$-states $\rightarrow s$-states of hydrogen) and thus lead to the decreasing of band gap value for about $0.4 \mathrm{eV}$.
Refractive indices dispersion of $\mathrm{K}_{1.75}\left(\mathrm{NH}_{4}\right)_{0.25} \mathrm{SO}_{4}$ single crystal for three crystal optics directions is deduced from calculated dielectric functions. Obtained curves reveal character close to experimentally established ones in visible spectrum region but lay somewhat lower (within $5.5 \%)$. Calculation did not reveal the crossing of refractive indices curves in a near IR region in contradiction to statement made from experiment. Such discrepancies are assumed to occur due to neglect of IR absorption of crystal in the simulations and presence of defects in real crystal.

\section{Acknowledgments}

Calculations have been performed using the Materials Studio 8.0 package at the supercomputer center of Wrocław University of Technology (WCSS). The work is based on the research provided by the grant support of the State Fund for Fundamental Research No.F76/36280 (2017).

\section{References}

[1] M.O. Romanyuk, B. Andriyevsky, O. Kostetsky, M.M. Romanyuk, V. Stadnyk, Condens. Matter Phys. 5, 586 (2002).

[2] M.O. Romanyuk, M.M. Romanyuk, Ferroelectrics 317, 57 (2005).

[3] P.A. Shchepanskyi, O.S. Kushnir, V.Yo. Stadnyk, A.O. Fedorchuk, M.Ya. Rudysh, R.S. Brezvin, P.Yu. Demchenko, A.S. Krymus, Ukr. J. Phys. Opt. 18, 187 (2017).

[4] C. González-Silgo, X. Solans, C. Ruiz-Pérez, M.L. Martínez-Sarrión, L. Mestres, E. Bocanegra, Condens. Matter 9, 2657 (1997).

[5] B. Andriyevsky, M. Jaskolski, V.Y. Stadnyk, M.O. Romanyuk, Z.O. Kashuba, M.M. Romanyuk, Computat. Mater. Sci. 79, 442 (2013).

[6] B. Andriyevsky, W. Ciepluch-Trojanek, V. Stadnyk, M. Tuzyak, M. Romanyuk, V. Kurlyak, J. Phys. Chem. Solids 68, 1892 (2007).

[7] O.V. Bovgyra, V.Y. Stadnyk, O.Z. Chyzh, Phys. Solid State 48, 1200 (2006).

[8] M.Ya. Rudysh, M.G. Brik, O.Y. Khyzhun, A.O. Fedorchuk, I.V. Kityk, P.A. Shchepanskyi, V.Yo. Stadnyk, G. Lakshminarayana, R.S. Brezvin, Z. Bak, M. Piasecki, RSC Adv. 7, 6889 (2017).

[9] M.Ya. Rudysh, V.Yo. Stadnyk, R.S. Brezvin, P.A. Shchepanskii, Condens. Matter Phys. 57, 50 (2015).

[10] A.Abu-El-Fadl, S. Bin Anooz, Cryst. Res. Technol. 41, 487 (2006).

[11] B.V. Andriyevsky, V.Yu. Kurlyak, N.A. Romanyuk, Bull. Acad. Sci. USSR Phys. Ser. 53, 1335 (1989).

[12] Castep Guide, materials studio 8.0, BIOVIA, Dassault Systèmes, 2014.

[13] Sonali Saha, T.P. Sinha, Abhijiti Mookerjee, Phys. Rev. B 62, 8828 (2000). 